\title{
Vertex Triangle Free Detour Number of a Graph
}

\author{
S. Sethu Ramalingam, I. Keerthi Asir`and S. Athisayanathan
}

\begin{abstract}
The $x$-triangle free detour number $d n_{\Delta f_{x}}(G)$ of a connected graph $G$ is the minimum order of its $x$-triangle free detour sets and any $x$-triangle free detour set $S_{x} \subseteq V$ of order $d n_{\Delta f_{x}}(G)$ is a $x$-triangle free detour basis of $G$. A connected graph of order $n$ with vertex triangle free detour number $n-1$ or $n-2$ for every vertex is characterized. Certain general properties satisfied by the vertex triangle free detour sets are studied.
\end{abstract}

Keywords: Triangle free detour distance, Triangle free detour number, Vertex triangle free detour set, Vertex triangle free detour number

Mathematics Subject Classification (2010): 05 C12

\section{Introduction}

The concept of triangle free detour distance was introduced by Keerthi Asir and Athisayanathan.[3] A path $P$ is called a triangle free path if no three vertices of $P$ induce a triangle. For vertices $u$ and $v$ in a connected graph $G$, the triangle free detour distance $D_{\Delta f}(u, v)$ is the length of a longest $u-v$ triangle free path in $G$. A $u-v$ path of length $D_{\triangle f}(u, v)$ is called a $u-v$ triangle free detour. The triangle free detour eccentricity $e_{\Delta f}(v)$ of a vertex in $\mathrm{G}$ is the maximum triangle free detour distance from $\mathrm{v}$ to a vertex of $\mathrm{G}$. The triangle free detour radius, $\operatorname{rad}_{\Delta f}(G)$ or $R_{\Delta f}$ of G is the minimum triangle free detour eccentricity among the vertices of $\mathrm{G}$, while the triangle free detour diameter, $\operatorname{diam}_{\Delta f}(G)$ or $D_{\Delta f}$ of $\mathrm{G}$ is the maximum triangle free detour eccentricity among the vertices of $\mathrm{G}$.

The concept of triangle free detour number was introduced and studied by Sethu Ramalingam et al. [6] A set $S \subseteq V$ is called a triangle free

\footnotetext{
*St. Xavier's College(Autonomous), Palayamkottai 627002; ssethusxc@gmail.com †St. Xavier's College(Autonomous), Palayamkottai 627002; asirsxc@gmail.com `St. Xavier’s College(Autonomous), Palayamkottai 627002; athisxc@gmail.com
}

Received: April 2016. Reviewed: May 2016 
detour set of $G$ if every vertex of $G$ lies on a triangle free detour joining a pair of vertices of $S$. The triangle free detour number $d n_{\Delta f}(G)$ of $G$ is the minimum order of its triangle free detour sets and any triangle free detour set of order $d n_{\Delta f}(G)$ is called a triangle free detour basis of $G$.

The concept of vertex detour number of a graph was introduced and studied in [4]. For any vertex $x$ in a connected graph $G$, a set $S$ of vertices of $G$ is an $x$-detour set if each vertex $v$ of $G$ lies on an $x-y$ detour in $G$ for some vertex $y$ in $S$. The minimum cardinality of an $x$-detour set of $G$ is defined as the $x$-detour number of $G$, denoted by $d_{x}(G)$ or simply $d_{x}$. An $x$-detour set of cardinality $d_{x}(G)$ is called a $d_{x}$-set of $G$. The concept of vertex detour monophonic number of a graph was in-

troduced and studied by Titus and Balakrishnan.[7] A chord of a path $P$ is an edge joining two non-adjacent vertices of $P$. A path $P$ is called monophonic if it is a chordless path. A longest $u-v$ monophonic path is called an $u-v$ detour monophonic path. For any vertex $x$ in a connected graph $G$, a set $S$ of vertices of $G$ is an $x$-detour monophonic set if each vertex $v$ of $G$ lies on an $x-y$ detour monophonic in $G$ for some vertex $y$ in $S$. The minimum cardinality of an $x$-detour monophonic set of $G$ is defined as the $x$-detour monophonic number of $G$, denoted by $d m_{x}(G)$ or simply $d m_{x}$. An $x$-detour monophonic set of cardinality $d m_{x}(G)$ is called a $d m_{x}$-set of $G$.

The concept of vertex geodetic number of a graph was introduced and studied by Santhakumaran et al. [5] For any vertex $x$ in a connected graph $G$, a set $S$ of vertices of $G$ is an $x$-geodetic set if each vertex $v$ of $G$ lies on an $x-y$ geodetic in $G$ for some vertex $y$ in $S$. The minimum cardinality of an $x$-geodetic set of $G$ is defined as the $x$-geodetic number of $G$, denoted by $g_{x}(G)$ or simply $g_{x}$. An $x$-geodetic set of cardinality $g_{x}(G)$ is called a $g_{x}$-set of $G$. Throughout this paper $G$ denotes a finite undirected simple connected graph with at least two vertices. For basic definitions and terminologies, we refer to Chartrand and Zhang. [1]

The following theorems are useful for the results in this paper.

Theorem 1.1. [2] Let $v$ be a vertex of a connected graph $G$. The following statements are equivalent:

(i) $v$ is a cut vertex of $G$.

(ii) There exist vertices $u$ and $w$ distinct from $v$ such that $v$ is on every $u-w$ path.

(iii) There exists a partition of the set of vertices $V-\{v\}$ into subsets $U$ and $W$ such that for any vertices $u \in U$ and $w \in W$, the vertex $v$ is on every $u-w$ path.

Theorem 1.2. [6] Every extreme-vertex of a connected graph $G$ belongs to every triangle free detour set of $G$.

Theorem 1.3. [6] If $T$ is a tree with $k$ end-vertices, then $d n_{\Delta f}(T)=k$. 
Theorem 1.4. [4] Let $x$ be any vertex of a connected graph $G$.

(i) Every end-vertex of $G$ other than the vertex $x$ (whether $x$ is end-vertex or not) belong to every $x$-detour set.

(ii) No cut vertex of $G$ belongs to any $d_{x}$-set.

Theorem 1.5. [7] Let $x$ be any vertex of a connected graph $G$.

(i) Every end-vertex of $G$ other than the vertex $x$ (whether $x$ is end-vertex or not) belong to every $x$-detour monophonic set.

(ii) No cut vertex of $G$ belongs to any $d m_{x}$-set.

Theorem 1.6. [5] Let $x$ be any vertex of a connected graph $G$.

(i) Every end-vertex of $G$ other than the vertex $x$ (whether $x$ is end-vertex or not) belong to every $x$-geodetic set.

(ii) No cut vertex of $G$ belongs to any $g_{x}$-set.

\section{Vertex Triangle Free Detour Number}

Let $x$ be a vertex of a connected graph $G$. A set $S_{x} \subseteq V$ is called an $x$ triangle free detour set of $G$ if every vertex $v$ of $G$ lies on a $x-y$ triangle free detour in $G$ for some vertex $y$ in $S_{x}$. The vertex triangle free detour number $d n_{\Delta f_{x}}(G)$ of $G$ is the minimum order of its $x$-triangle free detour sets and any $x$-triangle free detour set of order $d n_{\Delta f_{x}}(G)$ is a vertex triangle free detour basis of $G$. An $x$-triangle free detour set of cardinality $d n_{\Delta f_{x}}(G)$ is called a $d n_{\Delta f_{x}}$-set of $G$.

Theorem 2.1. For any vertex $x$ in $G, x$ does not belong to any $d n_{\Delta f_{x}}$-set of $G$.

Proof. Suppose that $x$ belongs to a $d n_{\Delta f_{x}}$-set, say $S_{x}$ of $G$. Since $G$ is a connected graph with at least two vertices, it follows from the definition of an $x$-triangle free detour set that $S_{x}$ contains a vertex $v$ different from $x$. Since the vertex $x$ lies on every $x-v$ triangle free detour in $G$, it follows that $T=S_{x}-\{x\}$ is an $x$-triangle free detour set of $G$, which is a contradiction to $S_{x}$ a minimum $x$-triangle free detour set of $G$.

Example 2.2. For the graph $G$ given in Figure 2.1, a minimum vertex triangle free detour sets and the vertex triangle free detour numbers are given in Table 2.1.

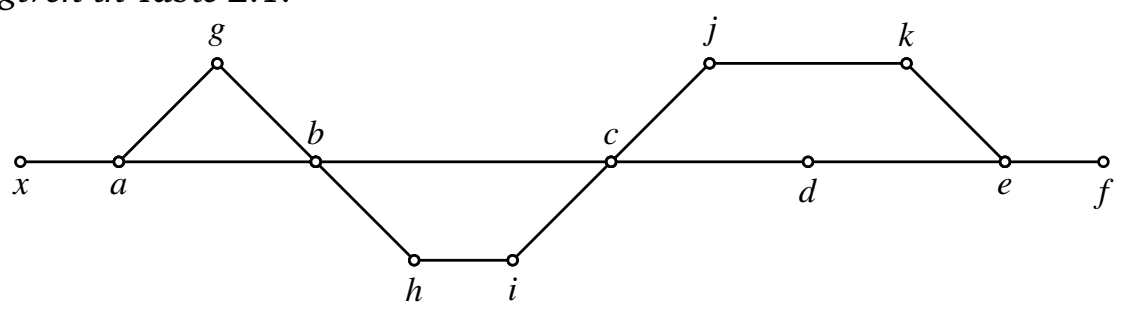


For the graph $G$ given in Figure 2.1, the sets $S_{1}=\{d, f\}, S_{2}=S_{1} \cup\{g\}$, $S_{3}=S_{2} \cup\{h\}$ and $S_{4}=S_{3} \cup\{j\}$ are minimum $x$-detour set, minimum $x$-triangle free detour set, minimum $x$-detour monophonic set and minimum $x$-geodetic set respectively and hence $d_{x}(G)=2, d n_{\Delta f_{x}}(G)=3$, $d m_{x}(G)=4$ and $g_{x}(G)=5$. Thus the vertex detour number, vertex triangle free detour number, vertex detour monophonic number and vertex geodetic number of a graph $G$ are distinct.

\begin{tabular}{ccc}
\hline Vertex $t$ & Minimum $d n_{\Delta f_{t}}$-set & $d n_{\Delta f_{t}}(G)$ \\
\hline$x$ & $\{g, d, f\}$ & 3 \\
$a$ & $\{x, g, d, f\}$ & 4 \\
$b$ & $\{x, g, d, f\}$ & 4 \\
$c$ & $\{x, g, d, f\}$ & 4 \\
$d$ & $\{x, g, f\}$ & 3 \\
$e$ & $\{x, g, d, f\}$ & 4 \\
$f$ & $\{x, g, d\}$ & 3 \\
$g$ & $\{x, d, f\}$ & 3 \\
$h$ & $\{x, g, d, f\}$ & 4 \\
$i$ & $\{x, g, d, f\}$ & 4 \\
$j$ & $\{x, g, d, f\}$ & 4 \\
$k$ & $\{x, g, d, f\}$ & 4 \\
\hline
\end{tabular}

Table 2.1

Remark 2.3. Let $x$ be any vertex of $G$. Then for any vertex $y$ belongs to $a d n_{\Delta f_{x}}$-set $S_{x}$ of $G$, the internal vertices of an $x-y$ triangle free detour may belong to $S_{x}$. For the graph $G$ given in Figure 2.2, $S_{x}=\{w, z\}$ is $a d n_{\Delta f_{x}}$-set of $G$ and $u$ belongs to $S_{x}$ is an internal vertex of the $x-w$ triangle free detour say $P: x, u, v, w$.

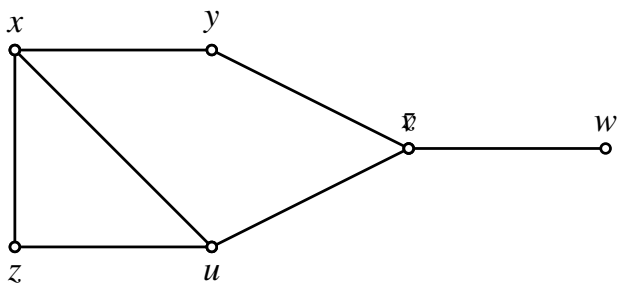

Figure 2.2: $G$

Theorem 2.4. Let $x$ be any vertex of a connected graph $G$.

(i) Every end-vertex of $G$ other than the vertex $x$ (whether $x$ is end-vertex or not) belong to every $x$-triangle free detour set.

(ii) No cut vertex of $G$ belongs to any $d n_{\triangle f_{x}}$-set. 
Proof. (i) Let $x$ be any vertex of $G$. By Theorem 2.1, $x$ does not belong to any $d n_{\Delta f_{x}}$-set. So let $v \neq x$ be an end-vertex of $G$. Then $v$ is the terminal vertex of an $x-v$ triangle free detour and $v$ is not an internal vertex of any triangle free detour so that $v$ belongs to every $x$-triangle free detour set of $G$.

(ii) Let $y$ be a cut vertex of $G$. Then by Theorem 1.1, there exists a partition of the set of vertices $V-\{y\}$ into subsets $U$ and $W$ such that for any vertex $u \in U$ and $w \in W$, the vertex $y$ is on every $u-w$ path. Hence, if $x \in U$, then for any vertex $w$ in $W, y$ lies on every $x-w$ path so that $y$ is an internal vertex of an $x-w$ triangle free detour. Let $S_{x}$ be any $d n_{\Delta f_{x}}$ - set of $G$. Suppose $S_{x} \cap \mathrm{W}=\phi$. Let $w_{1} \in W$. Since $S_{x}$ is an $x$-triangle free detour set, there exists an element $z$ in $S_{x}$ such that $w_{1}$ lies in some $x-z$ triangle free detour $P: x=z_{0}, z_{1}, \ldots, w_{1}, \ldots, z_{n}=z$ in $G$. Then the $x-w_{1}$ subpath of $P$ and $w_{1}-z$ subpath of $P$ both contain $y$ so that $P$ is not a path in $G$. Hence $S_{x} \cap \mathrm{W} \neq \phi$. Let $w_{2} \in S_{x} \cap W$. Then $y$ is an internal vertex of an $x-w_{2}$ triangle free detour. If $y \in S_{x}$, let $S=S_{x}-\{y\}$. It is clear that every vertex that lies on $x-y$ triangle free detour also lies on an $x-w_{2}$ triangle free detour. Hence it follows that $S$ is an $x$-triangle free detour set of $G$, which is a contradiction to $S_{x}$ is a minimum $x$-triangle free detour set of $G$. Thus $y$ does not belong to any $d n_{\Delta f_{x}}$-set. Similarly if $x \in W, y$ does not belong to any $d n_{\Delta f_{x}}$-set. If $x=y$, then by Theorem 2.1, $y$ does not belong to any $d n_{\Delta f_{x}}$-set.

Remark 2.5. If $x$ is an end-vertex of $G, x$ does not belong to any $d n_{\Delta f_{x}}{ }^{-}$ set by Theorem 2.1 .

Corollary 2.6. Let $T$ be a tree with $t$ end-vertices. Then $d n_{\Delta f_{x}}(T)=t-1$ or $d n_{\Delta f_{x}}(T)=t$ according to whether $x$ is an end-vertex or not. In fact, if $W$ is the set of all end-vertices of $T$, then $W-\{x\}$ is the unique $d n_{\Delta f_{x}}$-set of $T$.

Proof. Let $W$ be the set of all end-vertices of $T$. It follows from Theorem 2.1 and Theorem 2.4 that $W-\{x\}$ is the unique $d n_{\Delta f_{x}}$-set of $T$ for any end-vertex $x$ in $T$ and $W$ is the unique $d n_{\Delta f_{x}}$-set of $T$ for any cut vertex $x$ in $T$. Thus $W-\{x\}$ is the unique $d n_{\Delta f_{x}}$-set of $T$ for any vertex $x$ in $T$.

Theorem 2.7. For any hamiltonian graph $G, d n_{\triangle f_{x}}(G)=1$ for every vertex $x$ in $G$.

Proof. Let $C$ be a hamiltonian cycle of $G$. Let $x$ be any vertex of $G$ and let $y$ be any adjacent vertex of $x$ in $G$. Clearly every vertex of $G$ lies on a triangle free detour joining $x$ and $y$. Thus $d n_{\Delta f_{x}}(G)=1$ for every vertex $x$ in $G$.

Remark 2.8. The converse of Theorem 2.7 is false. For the graph $G$ given in Figure 2.3, $d n_{\Delta f_{x}}(G)=1$ for every vertex $x$ in $G$. But $G$ is not hamiltonian. 


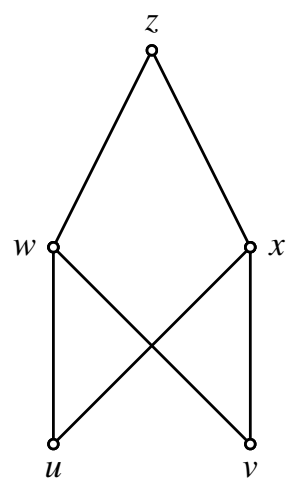

Figure 2.3: $G$

The following theorem is an easy consequence of the definition of the vertex triangle free detour number.

Theorem 2.9. (i) For any path $P_{n}, d n_{\Delta f_{x}}\left(P_{n}\right)=1$ or $d n_{\triangle f_{x}}\left(P_{n}\right)=2$ according as $x$ is an end-vertex or not.

(ii) For any cycle $C_{n}, d n_{\Delta f_{x}}\left(C_{n}\right)=1$ for every vertex $x$ in $C_{n}$.

(iii) For the wheel $W_{n}=K_{1}+C_{n-1}(n \geq 5), d n_{\Delta f_{x}}\left(W_{n}\right)=n-1$ or $d n_{\Delta f_{x}}\left(W_{n}\right)=$ 2 according as $x$ is in $K_{1}$ or $x$ in $C_{n-1}$.

(iv) For every vertex $x$ in $G, d n_{\Delta f_{x}}\left(K_{1, m}\right)=m$ or $d n_{\Delta f_{x}}\left(K_{n, m}\right)=m-1$ if $m \geq 2$.

(v) For any complete graph $K_{n}, d n_{\Delta f_{x}}\left(K_{n}\right)=n-1$ for every vertex $x$ in $K_{n}$.

(vi) For every vertex $x$ in $G, d n_{\Delta f_{x}}\left(K_{n, m}\right)=1$ if $n=m=1$.

Theorem 2.10. Let $G$ be a connected graph with cut vertices and let $S_{x}$ be an $x$-triangle free detour set of $G$. Then every branch of $G$ contains an element of $S_{x} \cup\{x\}$.

Proof. Suppose that there is a branch $B$ of $G$ at a cut vertex $v$ such that $B$ contains no vertex of $S_{x} \cup\{x\}$. Then clearly, $x \in V-\left(S_{x} \cup V(B)\right)$. Let $u \in V(B)-\{v\}$. Since $S_{x}$ is an $x$-triangle free detour set, there is an element $y \in S_{x}$ such that $u$ lies in some $x-y$ triangle free detour $P: x=u_{0}, u_{1}, \ldots, u, \ldots, u_{n}=y$ in $G$. By Theorem 1.1 the $x-u$ subpath of $P$ and $u-y$ subpath of $P$ both contain $v$, and it follows that $P$ is not a path, contrary to assumption.

Since every end-block $B$ is a branch of $G$ at some cut-vertex, it follows by Theorems 2.4 and 2.10 that every $d n_{\Delta f_{x}}$-set of $G$ together with the vertex $x$ contains at least one vertex from $B$ that is not a cutvertex. Thus the following corollaries are consequences of Theorem 2.10 . 
Corollary 2.11. If $G$ is a connected graph with $k$ end-blocks, then $d n_{\Delta f_{x}}(G) \geq k-1$ for every vertex $x$ in $G$.

Theorem 2.12. For any vertex $x$ in $G, 1 \leq d n_{\Delta f_{x}}(G) \leq n-1$.

Proof. It is clear from the definition of $d n_{\Delta f_{x}}$-set that $d n_{\Delta f_{x}}(G) \geq 1$. Also since the vertex $x$ does not belong to any $d n_{\Delta f_{x}}$-set, it follows that $d n_{\triangle f_{x}}(G) \leq n-1$.

Remark 2.13. The bounds in Theorem 2.13 are sharp. For the cycle $C_{n}$, $d n_{\Delta f_{x}}\left(C_{n}\right)=1$ for every vertex $x$ in $C_{n}$. Also for any path $P_{n}, d n_{\Delta f_{x}}\left(P_{n}\right)=1$ for any end-vertex $x$ in $P_{n}$. For the graph $K_{n}, d n_{\Delta f_{x}}\left(K_{n}\right)=n-1$ for every vertex $x$ in $K_{n}$.

In the following theorem, we establish the relationship between the vertex triangle free detour number of a graph of a vertex and the triangle free detour number of a graph.

Theorem 2.14. For any vertex $x$ in $G, 2 \leq d n_{\Delta f}(G) \leq d n_{\Delta f_{x}}(G)+1$.

Proof. A triangle free detour set needs at least two vertices so that $d n_{\Delta f}(G) \geq 2$. Let $x$ be any vertex of $G$ and let $S_{x}$ be a $d n_{\Delta f_{x}}$-set of $G$. Then every vertex of $G$ lies on an $x-y$ triangle free detour for some $y$ in $S_{x}$. Thus $S_{x} \cup\{x\}$ is a triangle free detour set of $G$. Since $d n_{\Delta f}(G)$ is the minimum cardinality of a triangle free detour set, it follows that $d n_{\Delta f}(G) \leq \operatorname{dn}_{\Delta f_{x}}(G)+1$.

Remark 2.15. The bound in Theorem 2.14 is sharp. For the complete graph $K_{n}, d n_{\Delta f}\left(K_{n}\right)=d n_{\Delta f_{x}}\left(K_{n}\right)+1$ for every vertex $x$ in $K_{n}$.

Theorem 2.16. For any two integers $a$ and $b$ with $2 \leq a \leq b+1$, there exists a connected graph $G$ with $d n_{\Delta f}(G)=a$ and $d n_{\Delta f_{x}}(G)=b$ for some vertex $x$ in $G$.

Proof. Case 1. $2 \leq a=b+1$. Let $G$ be any tree with $a$ end-vertices. By Theorem 1.3, $d n_{\Delta f}(G)=a$ and by Corollary 2.6, $d n_{\Delta f_{x}}(G)=b$ for an end-vertex $x$ in $G$.

Case 2. $2 \leq a<b+1$. Let $F=\left(P_{3} \cup P_{2} \cup(b-a+1) K_{1}\right)+\overline{K_{2}}$, where $U=V\left(P_{3}\right)=\left\{u_{1}, u_{2}, u_{3}\right\}, W=V\left(P_{2}\right)=\left\{w_{1}, w_{2}\right\}, X=V\left((b-a+1) K_{1}\right)=$ $\left\{x_{1}, x_{2}, \ldots, x_{b-a+1}\right\}$ and $V\left(\overline{K_{2}}\right)=\{x, y\}$. Let $G$ be the graph obtained from $F$ by adding $a-1$ new vertices $z_{1}, z_{2}, \ldots, z_{a-1}$ and joining each $z_{i}(1 \leq i \leq$ $a-1)$ to $u_{1}$. The graph $G$ is shown in Figure 2.4. Let $Z=\left\{z_{1}, z_{2}, \ldots, z_{a-1}\right\}$ be the set of end vertices of $G$. 


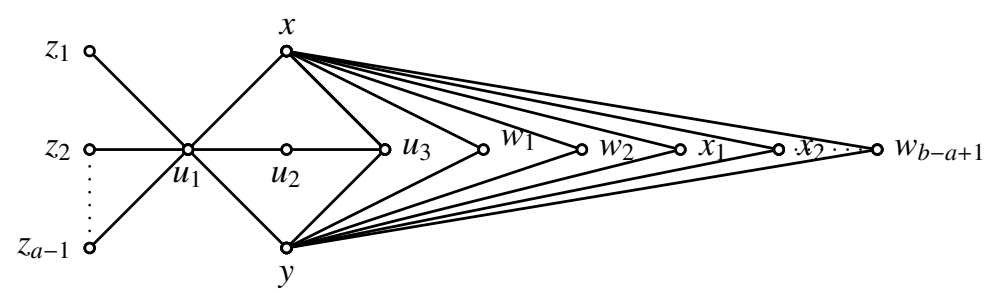

Figure 2.4: $G$

First, we show that $d n_{\Delta f}(G)=a$. By Theorem 1.2 , every triangle free detour set of $G$ contains $Z$. Since $Z \cup\left\{u_{1}\right\} \neq V(G)$, it follows that $Z$ is not a triangle free detour set of $G$ and so that $d n_{\Delta f}(G)>|Z|=a-1$. On the other hand, let $S=Z \cup\left\{w_{1}\right\}$. Then $D_{\Delta f}\left(z_{1}, w_{1}\right)=7$ and for each $i$ with $1 \leq i \leq b-a+1$, the path $z_{1}, u_{1}, u_{2}, u_{3}, y, x_{i}, x, w_{1}$ is a $z_{1}-w_{1}$ triangle free detour in $G$. Hence $S$ is a triangle free detour set of $G$ and so $d n_{\Delta f}(G) \leq|S|=a$. Therefore $d n_{\Delta f}(G)=a$.

Next we show that $d n_{\Delta f_{x}}(G)=b$ for the vertex $x$. Let $S_{x}$ be a minimum $x$-triangle free detour set of $G$. By Theorem 2.5 (i), $Z \subseteq S_{x}$. Since $D_{\Delta f}(x, Z)=6$ and no $x_{i}(1 \leq i \leq b-a+1)$ lies on an $x-z$ triangle free detour for any $z \in Z, Z$ is not an $x$-triangle free detour set of $G$. Now we claim that $X \subseteq S_{x}$. Assume, to the contrary, $X \notin S_{x}$. Then there exists an $x_{i}$ such that $x_{i} \notin S_{x}(1 \leq i \leq b-a+1)$. Now this $x_{i}$ does not lie on any $x-v$ triangle free detour for $v \neq x_{i}$ and $v \in S_{x}$, this is a contradiction to $S_{x}$ is a $x$-triangle free detour basis. Thus $X \subseteq S_{x}$. It is clear that $X \cup Z$ is an $x$-triangle free detour set. Hence it follows that $X \cup Z$ is an $x$-triangle free detour basis so that $d n_{\Delta f_{x}}(G)=a-1+b-a+1=b$.

\section{Bounds for the Vertex Triangle Free Detour Number of a Graph}

Theorem 3.1. For any vertex $x$ in a connected graph $G$ of order $n$ and a triangle free detour eccentricity $e_{\Delta f}(x), d n_{\Delta f_{x}}(G) \leq n-e_{\Delta f}(x)$.

Proof. Let $x$ be any vertex of $G$ and $v$ a triangle free detour eccentric vertex of $x$. Then $D_{\Delta f}(u, v)=e_{\Delta f}(x)$. Let $P: x=x_{0}, x_{1}, \ldots, x_{k}=v$ be an $x-v$ triangle free detour in $G$. Let $S=V(G)-\left\{x_{0}, x_{1}, \ldots, x_{k-1}\right\}$. Since each $x_{i}(0 \leq i \leq k-1)$ lies on an $x-v$ triangle free detour, $S$ is an $x$-triangle free detour set of $G$ so that $d n_{\Delta f_{x}}(G) \leq n-e_{\Delta f}(x)$.

Remark 3.2. The bounds in Theorem 3.1 is sharp. For the cycle $C_{n}$, $d n_{\Delta f_{x}}\left(C_{n}\right)=1=n-e_{\Delta f}(x)$ for every vertex $x$ in $C_{n}$. Also for the graph $G$ in Figure 3.1, $n=10, e_{\Delta f}\left(x_{7}\right)=7$ and $S=\left\{x_{4}, x_{9}, x_{10}\right\}$ is a $d n_{\Delta f_{x 7}}$ set so that $d n_{\Delta f x_{7}}=3$. Thus $d n_{\Delta f x_{7}}=n-e_{\Delta f}\left(x_{7}\right)$. The inequality in Theorem 3.1 can also be strict. For the same graph $G$ given in Figure 3.1, $e_{\Delta f}\left(x_{3}\right)=5$ and $S=\left\{x_{4}, x_{7}, x_{9}, x_{10}\right\}$ is a $d n_{\Delta f x_{3}}$-set so that $d n_{\Delta f x_{3}}(G)=4$. Thus $d n_{\Delta f x_{3}}(G)<n-e_{\Delta f}\left(x_{3}\right)$. 


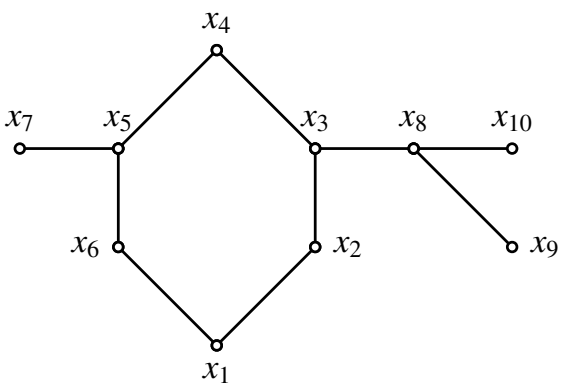

Figure 3.1: $G$

Corollary 3.3. If $G$ is a connected graph of order $n$ and triangle free detour diameter $D_{\Delta f}$, then $d n_{\Delta f_{x}}(G) \leq n-\frac{D_{\Delta f}}{2}$ for every vertex $x$ in $G$.

Proof. Since $R_{\Delta f} \leq e_{\Delta f}(x)$ for every vertex $x$ in $G$ and Theorem 3.1 that $d n_{\triangle f_{x}}(G) \leq n-\frac{D_{\Delta f}}{2}$.

Remark 3.4. The bound in Corollary 3.3 is sharp. For the star $K_{1, n-1}(n \geq$ 3), by Theorem 2.10(iv), $d n_{\Delta f_{x}}\left(K_{1, n}\right)=n-1=n-\frac{D_{\Delta f}}{2}$ for the cut vertex $x$ in $K_{1, n-1}$. Also, the inequality in Corollary 3.3 can be strict. For the star $K_{1, n-1}(n \geq 3)$, by Theorem $2.10(i v), d n_{\triangle f_{x}}\left(K_{1, n-1}\right)=n-2<n-\frac{D_{\Delta f}}{2}$ for an end vertex $x$ in $K_{1, n-1}$.

Theorem 3.5. Let $G$ be a connected graph of order $n \geq 2$ and $G \neq K_{3}$. Then $G=K_{1, n-1}$ if and only if $d n_{\Delta f_{x}}(G)=n-1$ or $d n_{\Delta f_{x}}(G)=n-2$ for every vertex $x$ of $G$.

Proof. If $G=K_{1, n-1}$, then by Theorem 2.10(iv), $d n_{\Delta f_{x}}(G)=n-1$ or $d n_{\triangle f_{x}}(G)=n-2$ for every vertex $x$ of $G$. If $n=2$, then $G=K_{2}=K_{1, n-1}$. If $n=3$, then $G=P_{3}=K_{1, n-1}$. Let $n \geq 4$. We prove that $G$ is a star. Suppose $G$ is not a star. If $G$ is a tree, then $G$ has at most $n-2$ endvertices. By Corollary 2.7, $d n_{\Delta f_{x}}(G) \leq n-3$ if $x$ is an end-vertex, which is a contradiction. Now, if $G$ is not a tree. Let $c(G)$ be the length of a longest cycle, say $C$ in $G$. If $c(G) \geq 4$, then $D_{\Delta f} \geq 3$ so that $e_{\Delta f}(x) \geq 3$ for some vertex $x$ in $G$. Hence by Theorem 3.1, $d n_{\Delta f_{x}}(G) \leq n-3$, which is a contradiction. If $c(G)=3$, let $u, v, w, u$ be a triangle in $G$. Since $n \geq 4$, there exists $x \in V(G)-\{u, v, w\}$ such that $x$ is adjacent to at least one of $u, v, w$ say $x u \in E(G)$. Then $x, u, v, w$ is a path in $G$ so that $e_{\Delta f}(x) \geq 3$. Then by Theorem 3.1, $d n_{\Delta f_{x}}(G) \leq n-3$, which is a contradiction. Thus $G$ is a star.

Theorem 3.6. Let $G$ be a connected graph of order $n \geq 5$. Then $d n_{\Delta f_{x}}(G)=n-2$ or $d n_{\Delta f_{x}}(G)=n-3$ for every vertex $x$ of $G$ if and only if $G$ is a double star or $K_{1, n-1}+e$.

Proof. It is straightforward to verify that if $G$ is a double star or $K_{1, n-1}+e$, then $d n_{\Delta f_{x}}(G)=n-2$ or $d n_{\Delta f_{x}}(G)=n-3$ for every vertex $x$ of $G$. For the converse, let $G$ be a connected graph of order $n \geq 5$ 
such that $d n_{\Delta f_{x}}(G)=n-2$ or $d n_{\Delta f_{x}}(G)=n-3$ for every vertex $x$ of $G$. If $D_{\Delta f} \leq 2$, then $G$ is the star $K_{1, n-1}$ and so by Theorem 2.10 (iv), $d n_{\Delta f_{x}}(G)=n-1$ for the cut vertex $x$ in $G$, which is a contradiction. Let $D_{\Delta f}=3$. If $G$ is a tree, then $G$ is a double star and the result follows from Corollary 2.7. Assume that $G$ is not a tree. Let $c(G)$ denote the length of a longest cycle in $G$. Since $D_{\Delta f}=3$, it follows that $c(G) \leq 4$. We consider two cases.

Case 1. Let $c(G)=4$. Let $C_{4}: v_{1}, v_{2}, v_{3}, v_{4}, v_{1}$ be a 4-cycle in $G$. Since $n \geq 5$ and $G$ is connected, there exists a vertex $x$ not on $C_{4}$ such that $x$ is adjacent to some vertex, say $v_{1}$ of $C_{4}$. Then $x, v_{1}, v_{2}, v_{3}, v_{4}$ is a path of length 4 in $G$ so that $D_{\Delta f} \geq 4$, which is a contradiction.

Case 2. Let $c(G)=3$. If $G$ contains two or more triangles, then $c(G)=4$ or $D_{\Delta f} \geq 4$, which is a contradiction. Hence $G$ contains an unique triangle $C_{3}: v_{1}, v_{2}, v_{3}, v_{1}$. Now, we prove that there is exactly one vertex on $C_{3}$ of degree at least 3 . If there are two or more vertices of $C_{3}$ having degree 3 or more, then $D_{\Delta f} \geq 4$, which is a contradiction. Thus exactly one vertex in $C_{3}$ has degree 3 or more. Since $D_{\Delta f}=3$, it follows that $G=K_{1, n-1}+e$. Now, it follows from Theorem 2.4 and Theorem 2.10 that $d n_{\Delta f_{x}}(G)=n-2$ or $d n_{\Delta f_{x}}(G)=n-3$ according as $x$ is a cut vertex or not. If $D_{\Delta f} \geq 4$, then $e_{\Delta f}(x) \geq 4$ for some vertex $x$ in $G$. Hence by Theorem 3.1, $d n_{\Delta f_{x}}(G) \leq n-e_{\Delta f}(x) \leq n-4$, which is a contradiction.

Remark 3.7. Theorem 3.6 is not true for $n=4$. For the graph $C_{4}, n=4$ and $d n_{\triangle f_{x}}(G)=1=n-3$ for every vertex $x$ in $G$. However, $G$ is neither a double star nor $K_{1, n-1}+e$.

Theorem 3.8. For every tree $T$ with triangle free detour diameter $D_{\Delta f}$, $d n_{\Delta f_{x}}(G)=n-D_{\Delta f}$ or $d n_{\Delta f_{x}}(G)=n-D_{\Delta f}+1$ for every vertex $x$ of $T$ if and only if $T$ is a caterpillar.

Proof. If $T$ be any tree. Let $P: u=u_{0}, v_{1}, \ldots, v_{D_{\Delta f}}=v$ be a triangle free detour diametral path. Let $k$ be the number of end vertices of $T$ and $l$ be the number of internal vertices of $T$ other than $v_{1}, v_{2}, \ldots, v_{D_{\Delta f}-1}$. Then $D_{\Delta f}-1+l+k=n$. By Corollary 2.6, $d n_{\Delta f_{x}}(T)=k$ or $d n_{\Delta f_{x}}(T)=k-1$ for every vertex $x$ of $T$ and so $d n_{\Delta f_{x}}(T)=n-D_{\Delta f}-1+l$ or $d n_{\Delta f_{x}}(T)=$ $n-D_{\Delta f}-1$ for every vertex $x$ of $T$. Hence $d n_{\Delta f_{x}}(T)=n-D_{\Delta f}+l$ or $d n_{\Delta f_{x}}(T)=n-D_{\Delta f}$ for every vertex $x$ of $T$ if and only if $l=0$ if and only if all the internal vertices of $T$ lie on the triangle free detour diametral path $P$ if and only if $T$ is a caterpillar.

Theorem 3.9. For every pair $a, b$ of integers with $1 \leq a \leq b$, there exists a connected graph $G$ with $d_{x}(G)=a$ and $d n_{\Delta f_{x}}(G)=b$. 
Proof. Case 1. For $1 \leq a=b$, any tree with $a$ end vertices has the desired properties, by Theorem 2.5 and Corollary 2.7 .

Case 2. For $1 \leq a<b$. Let $P_{i}: v_{i}(1 \leq i \leq b-a)$ be a $b-a$ copies of a path of order 1 and $P: x, u_{1}, u_{2}, u_{3}$ a path of order 4 . Let $G$ be the graph obtained by joining each $v_{i}(1 \leq i \leq b-a)$ in $P_{i}$ and $u_{1}$ in $P$ and $u_{2}$ in $P$. Adding $a$ new vertices $w_{1}, w_{2}, \ldots, w_{a}$ and joining each $w_{i}(1 \leq i \leq a)$ to $u_{3}$. The resulting graph $G$ of order $b+4$ is shown in Figure 3.2. Let $S_{1}=\left\{x, w_{1}, w_{2}, \ldots, w_{a}\right\}$ be the set of all extreme vertices of $G$. It is easily verified that $S=S_{1}-\{x\}$ is a $x$-detour set of $G$ and so by Theorem 1.4, $d_{x}(G)=|S|=a$.

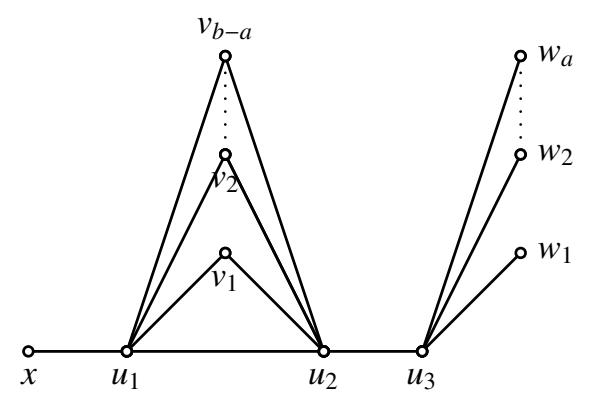

Figure 3.2: $G$

Next, we show that $d n_{\Delta f_{x}}(G)=b$. By Theorem 2.4, every $x$-triangle free detour set of $G$ contains $S$. Clearly, $S$ is not a triangle free detour set of $G$. It is easily verified that each $v_{i}(1 \leq i \leq b-a)$ must belong to every $x$-triangle free detour set of $G$. Thus $T=S \cup\left\{v_{1}, v_{2}, \ldots, v_{b-a}\right\}$ is a $x$-triangle free detour set of $G$, it follows from Theorem 2.4 that $T$ is a $x$-triangle free detour basis of $G$ and so $d n_{\Delta f_{x}}(G)=b$.

Theorem 3.10. For every pair $a, b$ of integers with $1 \leq a \leq b$, there exists a connected graph $G$ with $d n_{\Delta f_{x}}(G)=a$ and $d m_{x}(G)=b$.

Proof. Case 1. For $1 \leq a=b$, any tree with $a$ end vertices has the desired properties, by Theorem 2.4 and Corollary 2.6.

Case 2. For $1 \leq a<b$. Let $P_{i}: s_{i}, t_{i}(1 \leq i \leq b-a)$ be a $b-a$ copies of a path of order 2 and $P: x, u_{1}, u_{2}, u_{3}$ a path of order 4 . Let $G$ be the graph obtained by joining each $s_{i}(1 \leq i \leq b-a)$ in $P_{i}$ to $u_{1}$ in $P$ and joining each $t_{i}(1 \leq i \leq b-a)$ in $P_{i}$ to $u_{2}$ in $P$. Adding $a$ new vertices $w_{1}, w_{2}, \ldots, w_{a}$ and joining each $w_{i}(1 \leq i \leq a)$ to $u_{3}$. The resulting graph $G$ of order $2 b-a+4$ is shown in Figure 3.3. Let $S_{1}=\left\{x, w_{1}, w_{2}, \ldots, w_{a}\right\}$ be the set of all extreme vertices of $G$. It is easily verified that $S=S_{1}-\{x\}$ is a $x$ trianlge free detour set of $G$ and so by Theorem 2.4, $d n_{\Delta f_{x}}(G)=|S|=a$. 


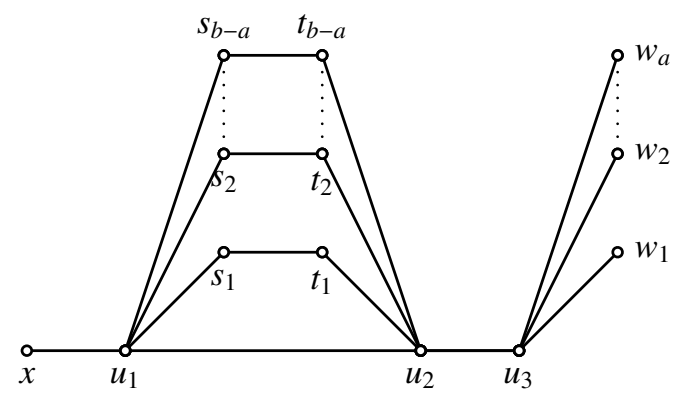

Figure 3.3: $G$

Next, we show that $d m_{x}(G)=b$. By Theorem 1.5, every $x$-detour monophonic set of $G$ contains $S$. Clearly, $S$ is not a detour monophonic set of $G$. It is easily verified that each $s_{i}(1 \leq i \leq b-a)$ or each $t_{i}(1 \leq i \leq b-a)$ must belong to every $x$-detour monophonic set of $G$. Thus $T=S \cup\left\{s_{1}, s_{2}, \ldots, s_{b-a}\right\}$ is a $x$-detour monophonic set of $G$, it follows from Theorem 2.5 that $T$ is a $x$-detour monophonic basis of $G$ and so $d m_{x}(G)=b$.

Theorem 3.11. For every pair $a, b$ of integers with $1 \leq a \leq b$, there exists a connected graph $G$ with $d n_{\Delta f_{x}}(G)=a$ and $g_{x}(G)=b$.

Proof. This follows from Theorem 3.10.

Theorem 3.12. For positive integers $a, b$ and $c \geq 2$ with $a<b$, there exists a connected graph $G$ with $R_{\Delta f}(G)=a, D_{\Delta f}(G)=b$ and $d n_{\Delta f_{x}}(G)$ $=c$ or $d n_{\Delta f_{x}}(G)=c-1$ for every vertex $x$ of $G$.

Proof. If $a=1$, then $b=2$. Take $G=K_{1, c}$. Then by Theorem 2.9(iv), $d n_{\triangle f_{x}}(G)=c$ or $d n_{\triangle f_{x}}(G)=c-1$ for every vertex $x$ of $G$. Now, let $a \geq 2$. We construct a graph $G$ with the desired properties as follows.

Let $C_{a+1}: v_{1}, v_{2}, \ldots, v_{a+1}, v_{1}$ be a cycle of order $a+1$ and let $P_{b-a+1}$ : $u_{0}, u_{1}, \ldots, u_{b-a}$ be a path of order $b-a+1$. Let $H$ be a graph obtained from $C_{a+1}$ and $P_{b-a+1}$ by identifying $v_{1}$ in $C_{a+1}$ and $u_{0}$ in $P_{b-a+1}$. Now, add $c-2$ new vertices $w_{1}, w_{2}, \ldots, w_{c-2}$ to $H$ by joining each vertex $w_{i}(1 \leq i \leq c-2)$ to the vertex $u_{b-a-1}$ and obtain the graph $G$ of Figure 3.4. Now, $R_{\Delta f}=a, D_{\Delta f}=b$ and $G$ has $c-1$ end vertices.

Case 1. Let $a$ be even. If $a=2$, then $d n_{\Delta f_{x}}(G)=c$ or $d n_{\Delta f_{x}}(G)=c-1$ according as $x \in\left\{v_{1}, u_{1}, u_{2}, \ldots, u_{b-a-1}\right\}$ or $x \in\left\{v_{2}, v_{3}, u_{b-a}, w_{1}, w_{2}, \ldots, w_{c-2}\right\}$. If $a \geq 4$, then $d n_{\Delta f_{x}}(G)=c$ or $d n_{\Delta f_{x}}(G)=c-1$ according as $x \in$ $\left\{v_{1}, v_{3}, v_{4}, \ldots, v_{a}, u_{1}, u_{2}, \ldots, u_{b-a-1}\right\}$ or $x \in\left\{v_{2}, v_{a+1}, u_{b-a}, w_{1}, w_{2}, . ., w_{c-2}\right\}$. 
Case 2. Let $a$ be odd. If $a=3$, then $d n_{\Delta f_{x}}(G)=c$ or $d n_{\Delta f_{x}}(G)=c-1$ according as $x \in\left\{v_{1}, u_{1}, u_{2}, \ldots, u_{b-a-1}\right\}$ or $x \in\left\{v_{2}, v_{3}, v_{4}, u_{b-a}, w_{1}, w_{2}, \ldots, w_{c-2}\right\}$. If $a \geq 5$, then $d n_{\Delta f_{x}}(G)=c$ or $d n_{\Delta f_{x}}(G)=c-1$ according as $x \in$ $\left\{v_{1}, v_{3}, v_{4}, \ldots, v_{(a+1) / 2}, v_{(a+5) / 2}, \ldots, v_{a}, u_{1}, u_{2}, \ldots, u_{b-a-1}\right\}$ or $x \in\left\{v_{2}, v_{(a+3) / 2}\right.$, $\left.v_{a+1}, u_{b-a}, w_{1}, w_{2}, \ldots, w_{c-2}\right\}$. Thus $d n_{\Delta f_{x}}(G)=c$ or $d n_{\Delta f_{x}}(G)=c-1$ for every vertex $x$ of $G$.

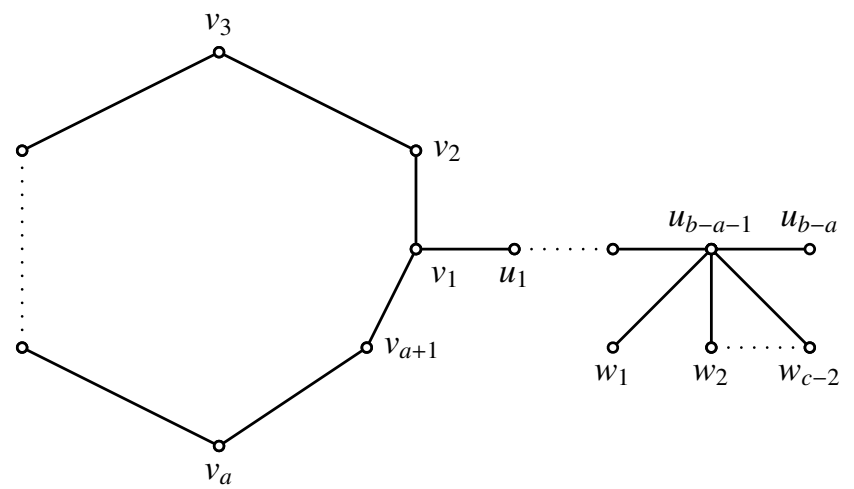

Figure 3.4: $G$

Theorem 3.13. For each triple $a, b$ and $n$ of positive integers with $1 \leq$ $b \leq n-a+1$ and $a \geq 4$, there exists a connected graph $G$ of order $n$ with triangle free detour diameter $D_{\Delta f}=a$ and $d n_{\Delta f_{x}}(G)=b$ or $d n_{\Delta f_{x}}(G)=$ $b-1$ for every vertex $x$ of $G$.

Proof. Let $G$ be a graph obtained from the cycle $C_{a}: u_{1}, u_{2}, \ldots, u_{a}, u_{1}$ of order $a$ by (i) adding $b-1$ new vertices $v_{1}, v_{2}, \ldots, v_{b-1}$ and joining each vertex $v_{i}(1 \leq i \leq b-1)$ to $u_{1}$ and (ii) adding $n-a-b+1$ new vertices $w_{1}, w_{2}, \ldots, w_{n-a-b+1}$ and joining each vertex $w_{i}(1 \leq i \leq n-a-b+1)$ to both $u_{1}$ and $u_{3}$. The graph $G$ has order $n$ and triangle free detour diameter $a$ and is shown in Figure 3.5. If $b=1, d n_{\Delta f_{x}}(G)=b$ for every vertex $x$ in $G$. If $b \geq 2$, then we consider two cases.

Case 1. Let $a$ be even. If $a=4$, then $d n_{\Delta f_{x}}(G)=b$ or $d n_{\Delta f_{x}}(G)=b-1$ according as $x=u_{1}$ or $x \in\left\{u_{2}, u_{3}, u_{4}, v_{1}, v_{2}, \ldots, v_{b-1}, w_{1}, w_{2}, \ldots, w_{n-a-b+1}\right\}$. If $a \geq 6$, then $d n_{\Delta f_{x}}(G)=b$ or $d n_{\Delta f_{x}}(G)=b-1$ according as $x \in$ $\left\{u_{1}, u_{2}, \ldots, u_{a / 2}, u_{(a+4) / 2}, \ldots, u_{a-1}, w_{1}, \ldots, w_{c-a-b+1}\right\}$ or $x \in\left\{u_{(a+2) / 2}, u_{a}, v_{1}, v_{2}\right.$, $\left.v_{b-1}\right\}$.

Case 2. Let $a$ be odd. Clearly $d n_{\Delta f_{x}}(G)=b$ or $d n_{\Delta f_{x}}(G)=b-1$ according as $x \in\left\{u_{1}, u_{2}, u_{2}, \ldots, u_{a-1}, w_{1}, \ldots, w_{n-a-b+1}\right\}$ or $x \in\left\{u_{a}, v_{1}, v_{2}, \ldots, v_{b-1}\right\}$. Thus $d n_{\Delta f_{x}}(G)=b$ or $d n_{\Delta f_{x}}(G)=b-1$ for every vertex $x$ of $G$. 


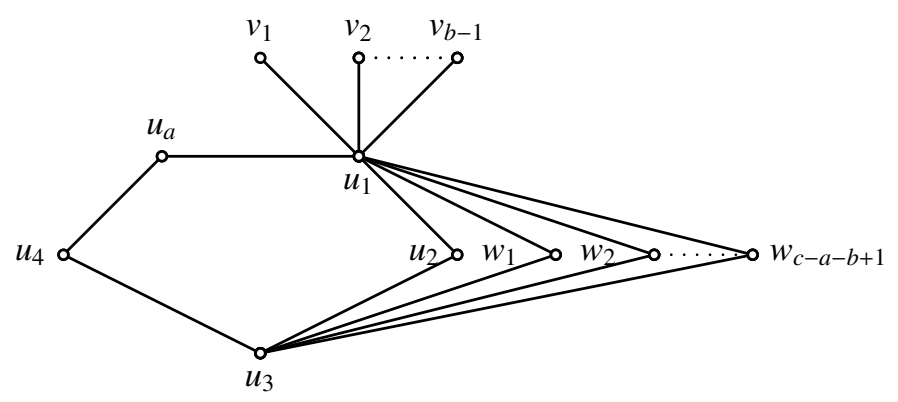

Figure 3.5: $G$

Theorem 3.14. Let $n \geq 2$ be any integer. For $1 \leq a \leq n-1$, there exists a connected graph $G$ with order $n$ and $d n_{\Delta f_{x}}(G)=a$ or $d n_{\triangle f_{x}}(G)=a-1$ for every vertex $x$ of $G$.

Proof. For $n=2, G=K_{2}$ has the desired properties. For $n=3, G=C_{3}$ or $P_{3}$ has the desired properties according as $a=1$ or $a=2$. For $n \geq 4$, we consider three cases.

Case 1. Let $a=1$. Then $G=C_{n}$ has the desired properties.

Case 2. Let $2 \leq a \leq n-2$. Then $n-a+1 \geq 3$. The graph $G$ is obtained from the cycle $C_{n-a+1}: u_{1}, u_{2}, \ldots, u_{n-a+1}, u_{1}$ by adding the $a-1$ new vertices $v_{1}, v_{2}, \ldots, v_{a-1}$ and joining these to $u_{1}$. The graph $G$ is shown in Figure 3.6.

Subcase a. Let $n-a+1$ be even. If $n-a+1=4$, then $d n_{\Delta f_{x}}(G)=a$ or $d n_{\Delta f_{x}}(G)=a-1$ according as $x=u_{1}$ or $x \in\left\{u_{2}, u_{3}, u_{4}, v_{1}, v_{2}, \ldots, v_{a-1}\right\}$. If $n-a+1 \geq 6$, then $d n_{\Delta f_{x}}(G)=a$ or $d n_{\Delta f_{x}}(G)=a-1$ according as $x \in\left\{u_{1}, u_{3}, u_{4}, \ldots, u_{(n-a+1) / 2}, u_{(n-a+5) / 2}, \ldots, u_{n-a}\right\}$ or $x \in\left\{u_{2}, u_{(n-a+3) / 2}, u_{n-a+1}\right.$, $\left.v_{1}, v_{2}, \ldots, v_{a-1}\right\}$.

Subcase b. Let $n-a+1$ be odd. If $n-a+1=3$, then $d n_{\triangle f_{x}}(G)=a$ or $d n_{\Delta f_{x}}(G)=a-1$ according as $x=u_{1}$ or $x \in\left\{u_{2}, u_{3}, v_{1}, v_{2}, \ldots, v_{a-1}\right\}$. If $n-a+1 \geq 5$, then $d n_{\Delta f_{x}}(G)=a$ or $d n_{\Delta f_{x}}(G)=a-1$ according as $x \in\left\{u_{1}, u_{3}, u_{4}, \ldots, u_{n-a}\right\}$ or $x \in\left\{u_{2}, u_{n-a+1}, v_{1}, v_{2}, \ldots, v_{a-1}\right\}$. Thus $d n_{\Delta f_{x}}(G)=a$ or $d n_{\Delta f_{x}}(G)=a-1$ for every vertex $x$ of $G$.

Case 3. Let $a=n-1$. Then $G=K_{1, n-1}$ has the desired properties. 


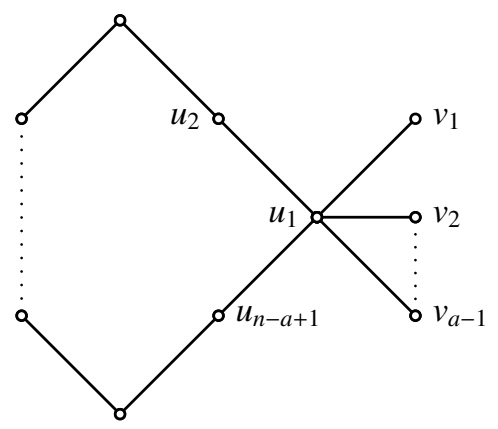

Figure 3.6: $G$

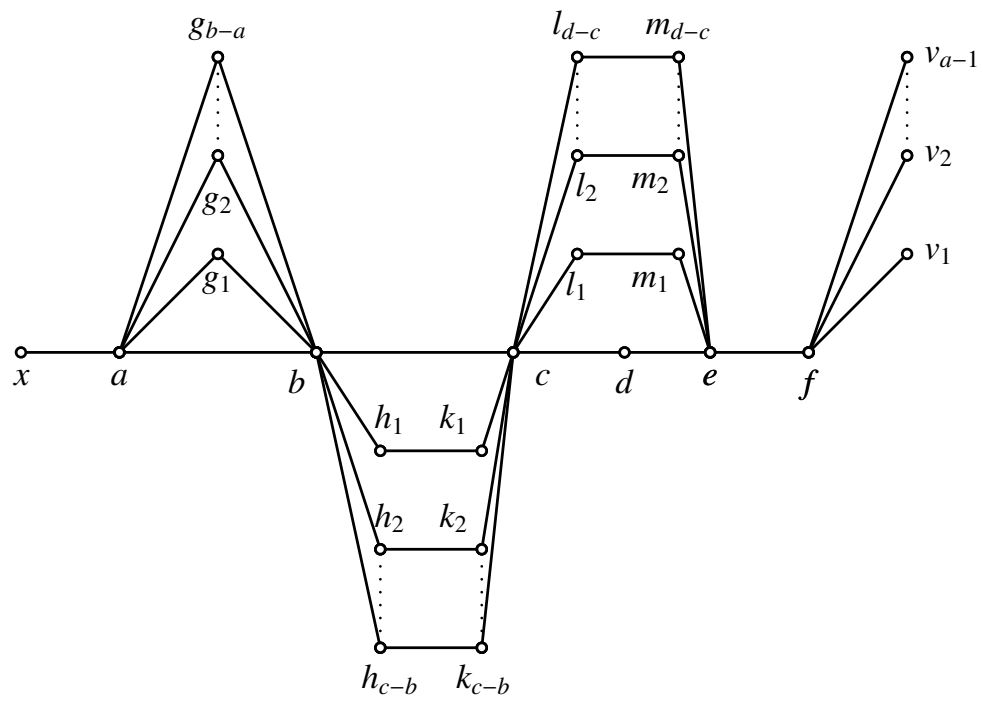

Figure 3.7: $G$

Theorem 3.15. For any four positive integers $a, b, c$ and $d$ of with $2 \leq a \leq b \leq c \leq d$, there exists a connected graph $G$ such that $d_{x}(G)=a$, $d n_{\triangle f_{x}}(G)=b, d m_{x}(G)=c$ and $g_{x}(G)=d$.

Proof. Let $2 \leq a \leq b \leq c \leq d$. Let $P: x, a, b, c, d, e, f$ be a path of order 7 and adding $a-1$ new vertices $v_{1}, v_{2}, v_{3}, v_{4}, \ldots . ., v_{a-1}$ to $f$. Let $P_{i}: g_{i}(1 \leq$ $i \leq b-a)$ be a $b-a$ copies of $K_{1}$ and joining each $g_{i}(1 \leq i \leq b-a)$ in $P_{i}$ to $a$ and $b$ in $P$. Let $P_{j}: h_{j}, k_{j}(1 \leq j \leq c-b)$ be a $c-b$ copies of a path of length 2 and joining each $h_{j}(1 \leq j \leq c-b)$ in $P_{j}$ to $b$ in $P$ and joining each $k_{j}(1 \leq j \leq c-b)$ in $P_{j}$ to $c$ in $P$. Let $P_{k}: l_{k}, m_{k}(1 \leq k \leq d-c)$ be a 
$d-c$ copies of a path of order 2 and joining each $l_{k}(1 \leq k \leq d-c)$ in $P_{k}$ to $c$ in $P$ and joining $m_{k}(1 \leq k \leq d-c)$ in $P_{k}$ to $e$ in $P$. The resulting graph $G$ is shown in Figure 3.7.

It is easily verify that $S_{1}=\left\{d, v_{1}, v_{2}, \ldots . ., v_{a-1}\right\}$ is a minimum $x$ detour set, $S_{2}=S_{1} \cup\left\{g_{1}, g_{1}, g_{2}, \ldots . ., g_{b-a}\right\}$ is a $x$-triangle free detour basis, $S_{3}=S_{2} \cup\left\{h_{1}, h_{2}, h_{3}, \ldots . ., h_{c-b}\right\}$ is a minimum $x$-detour monophonic set and $S_{4}=S_{3} \cup\left\{l_{1}, l_{2}, l_{2}, \ldots . ., l_{d-c}\right\}$ is a minimum $x$-geodetic set. Thus $d n_{x}(G)=a, d n_{\Delta f_{x}}(G)=b, d m_{x}(G)=c$ and $g_{x}(G)=d$.

\section{References}

[1] G. Chartrand and P. Zhang, Introduction to Graph Theory. New Delhi: Tata McGraw-Hill, 2006.

[2] F. Harary, Graph Theory. Reading, MA: Addison-Wesley, 1969.

[3] I. Keerthi Asir and S. Athisayanathan, "Triangle Free Detour Distance in Graphs," J. Combin. Math. Combin. Comput., to be published.

[4] A. P. Santhakumaran and P. Titus, "The Vertex Detour Number of a Graph," AKCE J. Graphs. Combin., vol. 4, no. 1, pp. 99 - 112, 2007.

[5] A. P. Santhakumaran and P. Titus, "Vertex Geodomination Number in Graphs," Bulletin of Kerala Mathematics Association, vol. 2, no. 2, pp. 45-57, 2005.

[6] S. Sethu Ramalingam, I. Keerthi Asir and S. Athisayanathan, "Triangle Free Detour Number of a Graph," submitted for publication.

[7] P. Titus and P. Balakrishnan, "The Vertex Detour Monophonic Number of a Graph," submitted for publication. 